\title{
PROCEDIMENTO FUZZY APLICADO À AVALIAÇÃO DA INSALUBRIDADE EM ATIVIDADES AGRÍCOLAS
}

\section{TADAYUKI YANAGI JUNIOR ${ }^{1}$, LEONARDO SCHIASSI ${ }^{2}$, LUCAS H. P. ABREU ${ }^{3}$, JACKSON A. BARBOSA ${ }^{4}$, ALESSANDRO T. CAMPOS ${ }^{5}$}

\begin{abstract}
RESUMO: Diante do alto grau de mecanização a que as atividades agrícolas estão sendo submetidas, objetivou-se, com esta pesquisa, desenvolver um modelo fuzzy capaz de avaliar e classificar o nível de insalubridade em diversos ambientes de trabalho. O modelo desenvolvido tem como variáveis de entrada: o índice de bulbo úmido e temperatura de globo (IBUTG, ${ }^{\circ} \mathrm{C}$ ), o nível de ruído (dBA), a taxa de metabolismo $\left(\mathrm{W} \mathrm{m}^{-2}\right)$ e o tempo de descanso (\%) e, como variável de saída, o índice de bem-estar humano (IBEH). O método de inferência utilizado foi o de Mandani e, na defuzificacão, utilizou-se o método do centro de gravidade. O sistema de regras foi desenvolvido com base nas combinações das variáveis de entrada. Foram definidas 400 regras com pesos iguais a 1, sendo que, na elaboração das regras, um especialista da área foi consultado. Foram utilizados dados de campo visando a testar o sistema desenvolvido, e os resultados mostraram que a modelagem proposta é uma ferramenta promissora na determinação do IBEH, apresentando tempo de descanso ideal variando de $64,2 \%$ (motosserra, próximo ao ouvido do operador) até $25 \%$ (derriçadora, $20 \mathrm{~m}$ de distância do operador), sendo que, diante de um cenário predefinido do ambiente térmico e acústico, foi possível determinar o grau de bem-estar humano e o tempo de descanso ideal para cada equipamento avaliado.
\end{abstract}

PALAVRAS-CHAVE: bem-estar humano, modelagem matemática, sistema especialista.

\section{FUZZY PROCEDURE APPLIED TO EVALUATE THE INSALUBRITY LEVEL IN AGRICULTURE ACTIVITIES}

\begin{abstract}
Given the high degree of mechanization to which agricultural activities are being submitted, the objective of this research was to develop a fuzzy model able to evaluate and classify the insalubrity level in different work environments. The model developed has as input variables: wet bulb globe temperature index (WBGTI, $\left.{ }^{\circ} \mathrm{C}\right)$, noise level $(\mathrm{dBA})$, metabolic rate $\left(\mathrm{W} \mathrm{m}{ }^{-2}\right)$ and rest time (\%); and has as output variable the human well-being index (HWBI). The Mamdani inference method was used, and for the defuzzification, the Center of Gravity method was used. The system of rules was developed based on combinations of input variables. Four hundred rules were defined, with weights equal to one, considering that in the development of rules, a specialist was consulted. Field data were used in order to test the developed system. The results showed that the proposed model is a promising tool in determining the HWBI, presenting the ideal rest time ranging from $64.2 \%$ (chainsaw, near the operator's ear) to $25 \%$ (harvester, 20 meters away from the operator), and, given a pre-defined scenario of thermal and acoustic environment, it was possible to determine the degree of human well-being and the ideal rest time for each equipment evaluated.
\end{abstract}

KEYWORDS: human well-being, mathematical modeling, expert systems.

\footnotetext{
${ }^{1}$ Professor Associado, Universidade Federal de Lavras (UFLA), Departamento de Engenharia (DEG), Lavras - MG.

${ }^{2}$ Eng ${ }^{-}$Agrícola, Doutorando em Engenharia Agrícola na Universidade Federal de Lavras (UFLA), Departamento de Engenharia (DEG), Lavras - MG.

${ }^{3}$ Graduando em Engenharia Agrícola, Universidade Federal de Lavras (UFLA).

${ }^{4}$ Professor Adjunto, Universidade Federal de Lavras (UFLA), Departamento de Engenharia (DEG), Lavras - MG.

${ }^{5}$ Professor Adjunto, Universidade Federal de Lavras (UFLA), Departamento de Engenharia (DEG), Lavras - MG.

Recebido pelo Conselho Editorial em: 12-5-2011

Aprovado pelo Conselho Editorial em: 3-2-2012
} 


\section{INTRODUÇÃO}

Durante os últimos anos, o bem-estar dos trabalhadores passou a ser uma das preocupações nas indústrias e agroindústrias para o adequado desempenho das atividades. Ambientes insalubres além de causar extenuação física e nervosa nos trabalhadores, provoca também queda no rendimento e afastamentos por problemas de saúde.

Dentre as fontes causadoras de estresse nos trabalhadores de agroindústrias, destacam-se a exposição ao calor, o ruído, a vibração e a iluminação, sendo que a intensidade e o tempo de exposição a cada um desses fatores afetam seu desempenho. Outros fatores são idade, gênero e adaptação prévia ao ambiente de trabalho (CARVALHO, 2009).

No Brasil, para a avaliação da exposição ao calor, a NR-15 (BRASIL, 1990), em seu anexo $\mathrm{n}^{\mathrm{o}} 3$ (Limites de tolerância para exposição ao calor), indica o uso do índice de bulbo úmido e temperatura de globo (IBUTG, ${ }^{\circ} \mathrm{C}$ ).

Recentemente, outras variáveis têm sido estudadas como forma complementar àquelas relacionadas ao ambiente térmico, tal como os níveis de pressão sonora ao qual o trabalhador está sendo submetido durante a atividade (BAESSO et al., 2008; MION et al., 2009).

Para a avaliação da insalubridade por ruído em locais de trabalho, a consolidação das leis do trabalho, na Portaria 3.214, NR-15 (BRASIL, 1990), estabelece os limites de exposição ao ruído para trabalhadores brasileiros (Tabela 1). Em adição às variáveis previamente citadas, a taxa de metabolismo e o tempo de descanso influenciam no bem-estar do trabalhador agrícola.

Visando a quantificar a interação dessas variáveis no conforto do trabalhador, podem-se utilizar técnicas de modelagem computacional, como sistemas especialistas inteligentes, capazes de executar tarefas ou resolver problemas a partir de uma base de conhecimento. Os sistemas mais utilizados e testados são a lógica fuzzy e redes neurais artificiais.

A metodologia fuzzy tem sido utilizada em várias áreas, como bem-estar animal (PANDORFI et al., 2007), análises de custo de produção (NÄÄS et al., 2010), sistemas de monitoração para redes de transmissão de energia elétrica (ALMEIDA \& KAGAN, 2010), detecção de câncer (SILVEIRA et al., 2008) e detecção de cio em vacas leiteiras (FERREIRA et al., 2007; BRUNASSI et al., 2010).

Tendo em vista que a interação entre as variáveis IBUTG, nível de ruído, taxa de metabolismo e tempo de descanso influenciam no bem-estar do trabalhador agrícola, a aplicação da lógica fuzzy é uma alternativa interessante, pois possibilita a classificação do ambiente de trabalho, evidenciando situações de insalubridade e danos à saúde do trabalhador.

Diante do exposto, objetivou-se, com o presente trabalho, desenvolver um modelo fuzzy para predizer o nível de insalubridade de ambientes, auxiliando na redução das perdas e evitando a exposição excessiva do trabalhador agrícola ao ambiente insalubre.

\section{MATERIAL E MÉTODOS}

Para o desenvolvimento do sistema fuzzy, foram definidas como variáveis de entrada: o índice de bulbo úmido e a temperatura de globo (IBUTG, ${ }^{\circ} \mathrm{C}$ ), o nível de ruído (dBA), o grau de metabolismo $\left(\mathrm{W} \mathrm{m}^{-2}\right)$ e a porcentagem de descanso $(\%)$ em relação ao tempo trabalhado, sendo $8 \mathrm{~h}$ o limite máximo de jornada de trabalho estabelecido pela legislação brasileira NR-15 (BRASIL, 1990). Com base nas variáveis de entrada, o sistema fuzzy prediz a variável de saída: índice de bemestar humano (IBEH).

$\mathrm{Na}$ análise, utilizou-se o método de inferência de Mamdani, que traz como resposta um conjunto fuzzy originado da combinação dos valores de entrada com os seus respectivos graus de pertinência, através do operador mínimo e, em seguida, pela superposição das regras por meio do operador máximo (LEITE et al., 2010). A defuzificação foi feita utilizando o método do Centro de Gravidade (Centroide ou Centro de Área), que considera todas as possibilidades de saída, transformando o conjunto fuzzy originado pela inferência em valor numérico (LEITE et al., 2010). 


\section{Variáveis de entrada}

Considerando que as principais variáveis que influenciam o ambiente térmico, nos mais variados ambientes de trabalho, são temperatura de bulbo seco, temperatura de bulbo úmido, umidade relativa e radiação solar, optou-se em utilizar como variável de entrada o IBUTG $\left({ }^{\circ} \mathrm{C}\right)$, que é calculado utilizando-se dessas variáveis. Foram consideradas cinco faixas de classificação, sendo que estes limites foram definidos conforme a norma regulamentadora NR-15 (BRASIL, 1990), que contém as estimativas das taxas de metabolismo por tipo de atividade.

Em função do IBUTG, os limites de tolerância para o regime de trabalho intermitente foram definidos (Tabela 1).

TABELA 1. Limites de tolerância para exposição ao calor, em regime de trabalho intermitente, com períodos de descanso no próprio local de trabalho (IBUTG, ${ }^{\circ} \mathrm{C}$ ). Tolerance limits for heat exposure, during intermittent work regime with rest periods at the workplace (WBGTI, ${ }^{\circ}$ ).

\begin{tabular}{lccc}
\hline \multirow{2}{*}{ Regime de Trabalho } & \multicolumn{3}{c}{ Tipo de Atividade } \\
\cline { 2 - 4 } & Leve & Moderada & Pesada \\
\hline Trabalho contínuo & até 30,0 & até 26,7 & até 25,0 \\
45 min trabalho - 15 min descanso & 30,1 a 30,6 & 26,8 a 28,0 & 25,1 a 25,9 \\
30 min trabalho - 30 min descanso & 30,7 a 31,4 & 28,1 a 29,4 & 26,0 a 27,9 \\
15 min trabalho - 45 min descanso & 31,5 a 32,2 & 29,5 a 31,1 & 28,0 a 30,0 \\
Não é permitida a exposição sem alguma & acima de 32,2 & acima de 31,1 & acima de 30,0 \\
medida de controle &
\end{tabular}

Fonte: NR-15 (BRASIL, 1990)

Para o desenvolvimento dos conjuntos fuzzy para variável nível de ruído (dBA), foram utilizados dados da literatura, em que diversos autores classificam intervalos de ruído de acordo com sua influência no conforto humano (Tabela 2). Assim, são definidos cinco conjuntos para essa variável.

TABELA 2. Intervalos de níveis de ruído utilizados para o desenvolvimento dos conjuntos fuzzy. Ranges of noise levels used for the development of fuzzy sets.

\begin{tabular}{ccc}
\hline Nível de Ruído (dBA) & Classificação & Fonte \\
\hline$[0 ; 40]$ & Nenhum efeito & Adaptado de ROBIN (1987) \\
{$[40 ; 55]$} & Pequeno desconforto & Adaptado de ROBIN (1987) \\
{$[55 ; 70]$} & Estresse leve e desconforto & Adaptado de OMS (2001) \\
{$[70 ; 100]$} & Desgaste do organismo & Adaptado de OMS (2001) \\
{$[100 ; 140]$} & Danos no aparelho auditivo & Adaptado de ROBIN (1987) \\
\hline
\end{tabular}

Para a variável de entrada grau de metabolismo $\left(\mathrm{W} \mathrm{m}^{-2}\right)$, foram usadas quatro faixas de classificação, classificadas conforme metodologia proposta por COUTO (1987), como consumo energético para atividades de repouso, leve, moderada e pesada (Tabela 3).

O tempo de descanso $(\%)$ foi dividido em quatro faixas $(0-25 ; 0-50 ; 25-75 ; 50-75)$, classificado segundo a NR-15 (BRASIL, 1990).

Os intervalos admitidos para as variáveis de entrada estão graficamente representados pelas curvas de pertinência trapezoidais e triangulares (Figura 1), por representarem melhor a variação dos dados de entrada e por serem as mais usadas de acordo com a literatura (SCHIASSI et al., 2008; BARIN et al., 2010). 
TABELA 3. Taxas metabólicas por tipo de atividade. Metabolic rates by type of work activity.

Tipo de atividades Metabolismo $\left(\mathrm{W} \mathrm{m}^{-2}\right)$

Repouso

Sentado em repouso 100

Leve

Sentado, com movimento de braços e tronco

Sentado, com movimentação de braços e pernas

De pé, trabalho leve, em máquina ou bancada, principalmente com os

150

braços

Moderado

Sentado, com movimentos vigorosos de braços e pernas

De pé, trabalho moderado, em máquina ou bancada, com alguma

movimentação

Em movimento, trabalho moderado de levantar, empurrar

300

\section{Pesado}

Trabalho pesado

Trabalho intermitente de levantar, empurrar ou arrastar pesos

550

Fonte: Couto (1987)

A

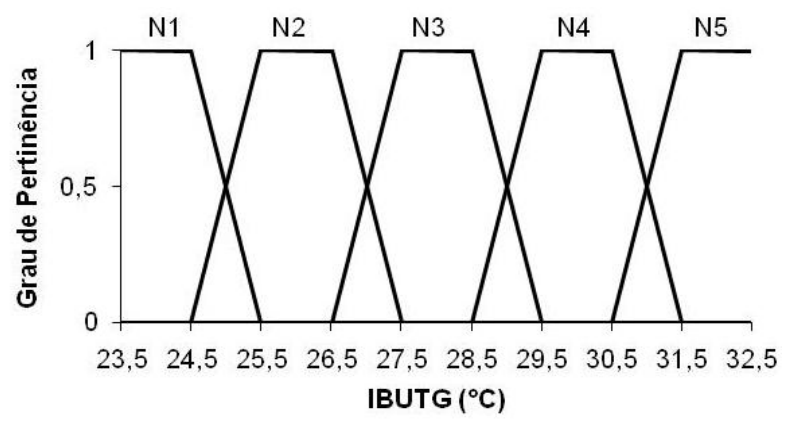

C

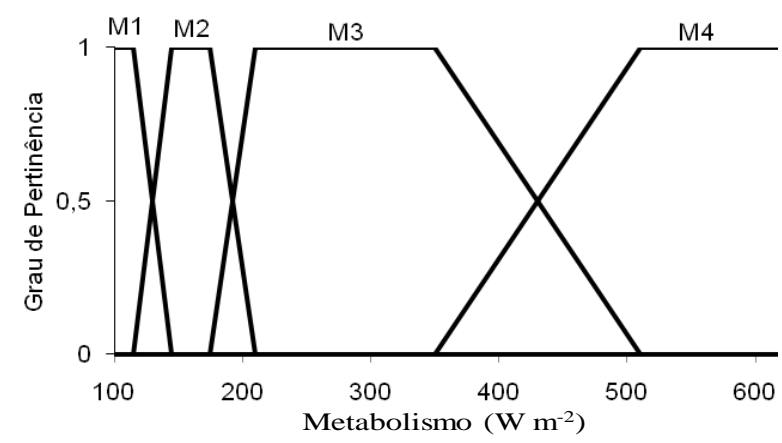

B

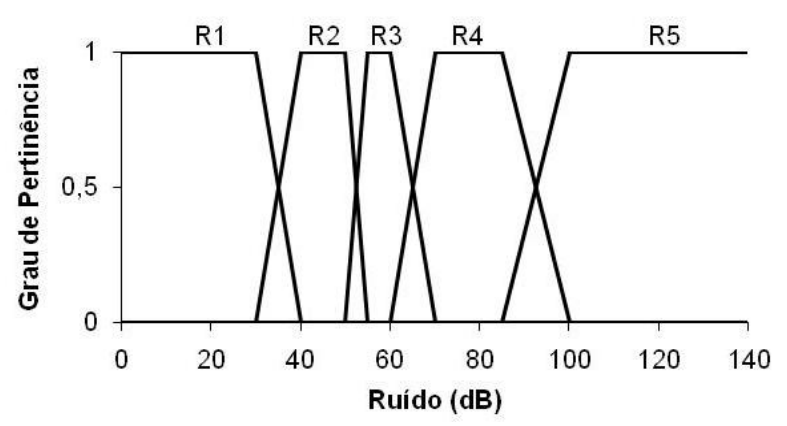

D

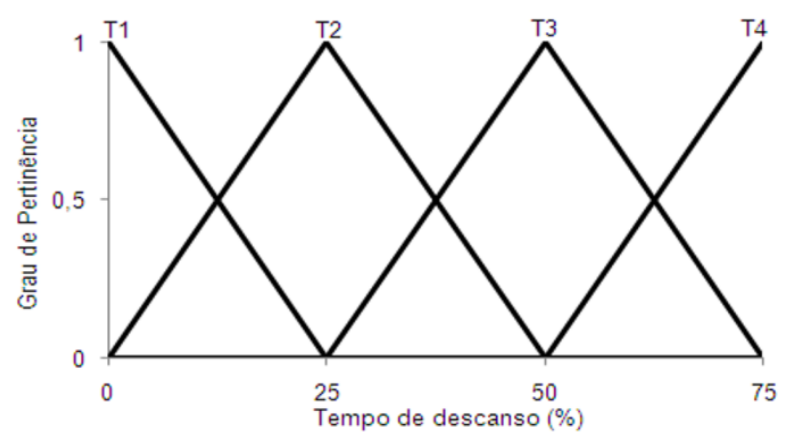

FIGURA 1. Funções de pertinência para as variáveis: A - IBUTG $\left({ }^{\circ} \mathrm{C}\right), \mathrm{B}$ - nível de ruído (dBA), C - grau de metabolismo $\left(\mathrm{W} \mathrm{m}^{-2}\right)$ e $\mathrm{D}$ - tempo de descanso $(\%)$. Pertinence functions for the variables: A - WBGTI $\left({ }^{\circ} \mathrm{C}\right), \mathrm{B}$ - noise level (dBA), C - metabolic rate

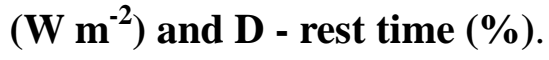

\section{Variável de saída}

A variável de saída índice de bem-estar humano (IBEH) permite a indicação direta do nível de bem-estar experimentado pelos trabalhadores. Seus conjuntos estabelecem intervalos no domínio de [0,1] e foram classificados segundo o mesmo conceito apresentado por OWADA et al. (2007) para o bem-estar de frangos de corte. Dessa forma, os seguintes conjuntos fuzzy para IBEH foram especificados, conforme ilustrado na Figura 2, considerando-se curvas de pertinência do tipo triangular. 


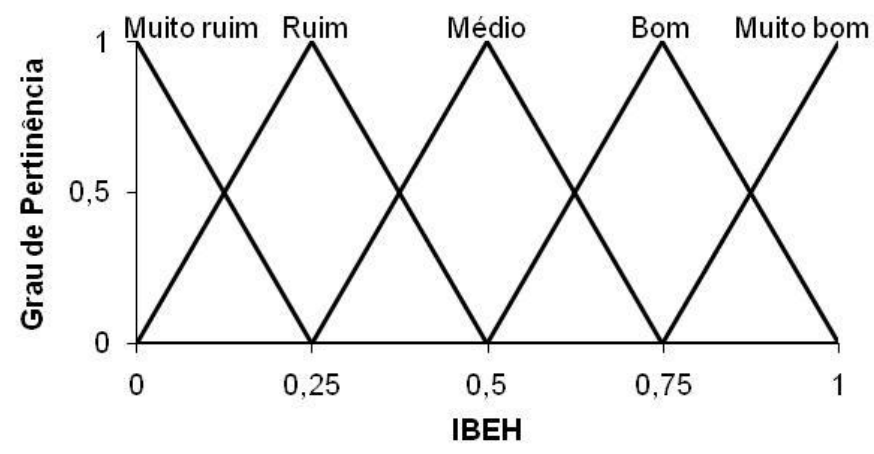

FIGURA 2. Funções de pertinência para a variável de saída índice de bem-estar humano (IBEH). Pertinence functions for the output variable human well-being index (HWBI).

\section{Sistema de regras}

O sistema de regras fuzzy foi criado com base em informações da literatura e por meio do auxílio de especialistas, em forma de sentenças linguísticas. Três especialistas foram selecionados conforme metodologia de seleção de especialista fuzzy proposta por CORNELISSEN et al. (2002) e utilizada por ESCOBAR \& GALINDO (2004). Dois especialistas possuem experiência em ambiência e um em mecanização, sendo que todos possuem mais de 10 anos de atuação nas respectivas áreas, caracterizando domínio sobre o tema. Esta característica é desejada de um especialista (AYYUB \& KLIR, 2006), tendo em vista sua influência direta na confiabilidade e na qualidade dos resultados (MARTINO, 1983; PREBLE, 1984; TAYLOR, 1988).

De acordo com as combinações dos dados de entrada, foram definidas 400 regras e, para cada regra, foi atribuído um fator de ponderação igual a 1 . O fator de ponderação igual a 1 , usualmente adotado como default, foi escolhido por mostrar-se adequado ao modelo proposto com base no comportamento dos resultados obtidos pelas simulações. Ademais, este valor tem sido adotado em diversos modelos fuzzy reportados pela literatura (FERREIRA et al., 2007; SCHIASSI et al., 2008; CREMASCO et al., 2010).

\section{Avaliações experimentais}

Dados de ruído e IBUTG foram coletados avaliando diferentes tipos de máquinas agrícolas, com o objetivo de testar o sistema fuzzy desenvolvido.

O experimento foi realizado no município de Lavras, Minas Gerais, com coordenadas geográficas de $21^{\circ} 14^{\prime} \mathrm{S}$ de latitude, $45^{\circ} 00^{\prime} \mathrm{W}$ de longitude, $918,8 \mathrm{~m}$ de altitude. Os dados foram coletados em área livre de obstáculos, com dimensões de 45,0 x 90,0 m, todo o entorno vegetado com bambu da espécie Bambusa vulgaris e solo coberto com grama-batatais.

As máquinas avaliadas foram: trator agrícola, motor modelo AGCO Sisu Power potência máxima de $62,5 \mathrm{~kW}$ em rotação de $2.200 \mathrm{rpm}$ e 3 cilindros aspirados; motosserra agropecuária, motor com potência máxima de $2,6 \mathrm{~kW}$ em rotação de $13.500 \mathrm{rpm}$ e $50,2 \mathrm{~cm}^{3}$ de cilindrada; roçadora costal motorizada, motor com potência máxima de $2,0 \mathrm{~kW}$ em rotação de $12.000 \mathrm{rpm}$ e 39 $\mathrm{cm}^{3}$ de cilindrada; derriçadora portátil, motor com potência máxima de $1,3 \mathrm{~kW}$ em rotação de $12.300 \mathrm{rpm}$ e $30,8 \mathrm{~cm}^{3}$ de cilindrada.

Os dados de temperatura de bulbo seco, temperatura de bulbo úmido e temperatura de globo negro, utilizados nos cálculos de IBUTG, foram coletados por sensores registradores (precisão de $\pm 3 \%$ ) fixados próximo ao operador, enquanto as leituras dos níveis de ruído foram realizadas na altura média do ouvido do operador e em circunferências equidistantes $2 \mathrm{~m}$ até o limite da área amostral de $20 \mathrm{~m}$. Em cada circunferência, foram distribuídos 12 sensores registradores igualmente espaçados (Figura 3), sendo que o tempo de coleta em cada circunferência foi de 1 min com intervalo de $1 \mathrm{~s}$ entre as coletas, totalizando, assim, 60 repetições por circunferência, conforme metodologia proposta por CUNHA \& TEODORO (2006) e MION et al. (2009). 


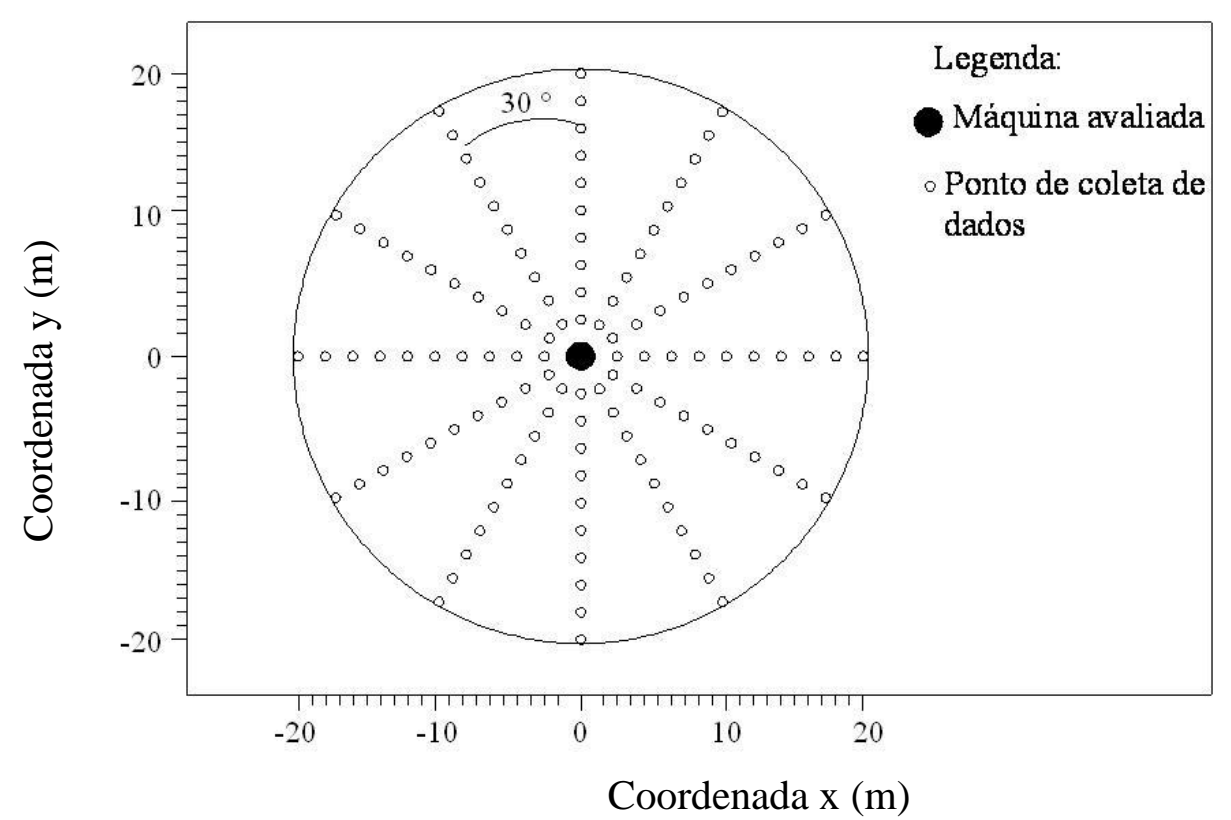

FIGURA 3. Croqui da área experimental. Lay-out of the experimental area.

As avaliações foram realizadas conforme metodologia descrita na NBR-9999 (ABNT, 1987), em que a temperatura ambiente deve estar entre -5 e $30^{\circ} \mathrm{C}$, e a velocidade do vento deve ser inferior a $5,0 \mathrm{~m} \mathrm{~s}^{-1}$.

A análise foi desenvolvida com o uso do fuzzy logic toolbox do MATLAB ${ }^{\circledR} 6.5$ (AMENDOLA et al., 2005), e o método de inferência utilizado foi o de Mamdani, conforme proposto por SIVANANDAM et al. (2007).

\section{Simulações e teste do modelo}

As simulações foram feitas por meio do modelo fuzzy desenvolvido, considerando como variáveis de entrada IBUTG, ruído, metabolismo e tempo de descanso.

Para testar o modelo fuzzy, foram utilizados dados experimentais de IBUTG médio durante o dia de coleta, em cada equipamento (trator $-21,8{ }^{\circ} \mathrm{C}$, motosserra $-20,9{ }^{\circ} \mathrm{C}$, roçadora $-22,6{ }^{\circ} \mathrm{C}$ e derriçadora $-22,1^{\circ} \mathrm{C}$ ) e nível de ruído médio para cada distância de afastamento, sendo simulado o tempo de descanso ideal para cada máquina, tendo como variáveis predefinidas do sistema o índice de bem-estar humano médio para atividade pesada, com consumo metabólico de $400 \mathrm{~W} \mathrm{~m}^{-2}$ (GOSLING \& ARAÚJO, 2008).

Nos testes desenvolvidos, foi simulado o tempo de descanso ideal em cada equipamento diante dos níveis de ruído e IBUTG encontrados nas avaliações de campo. Esses testes tiveram por objetivo avaliar a capacidade do modelo proposto em predizer uma situação de estresse em atividades agrícolas e propor um tempo de descanso ideal para que o trabalhador não esteja sujeito a problemas de saúde advindos da atividade exercida, além de evitar situações de insalubridade ao trabalhador.

\section{RESULTADOS E DISCUSSÃO}

\section{Desenvolvimento do modelo}

Para análise do sistema fuzzy desenvolvido, foram utilizados gráficos tridimensionais, sendo essa a forma mais conhecida de representação dos resultados simulados em modelos fuzzy (OWADA et al., 2007; PEREIRA et al., 2008). As superfícies ilustradas na Figura 4 mostram as variações de IBEH para as diversas combinações das variáveis de entrada. Nessa simulação, as variáveis de entrada foram prefixadas em intervalos de conforto (IBUTG $=23,5^{\circ} \mathrm{C}$, nível de ruído $=$ $50 \mathrm{dBA}$, tempo de descanso $=25 \%$ e metabolismo $=400 \mathrm{~W} \mathrm{~m}^{-2}$ ) para exercício de uma atividade pesada, conforme classificação da NR-15 (BRASIL, 1990). 
A

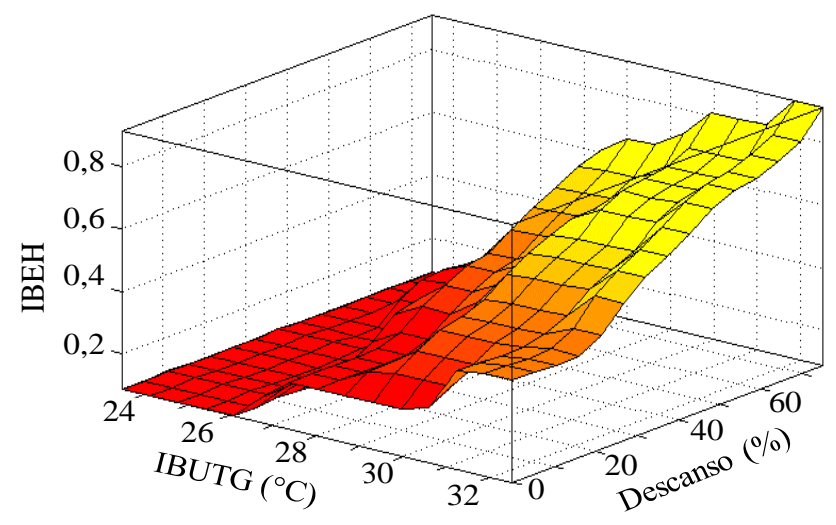

$\mathrm{C}$

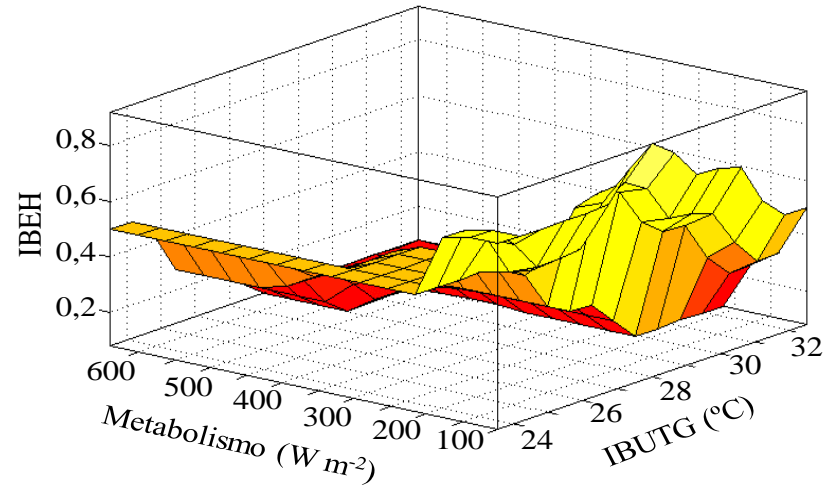

$\mathrm{E}$

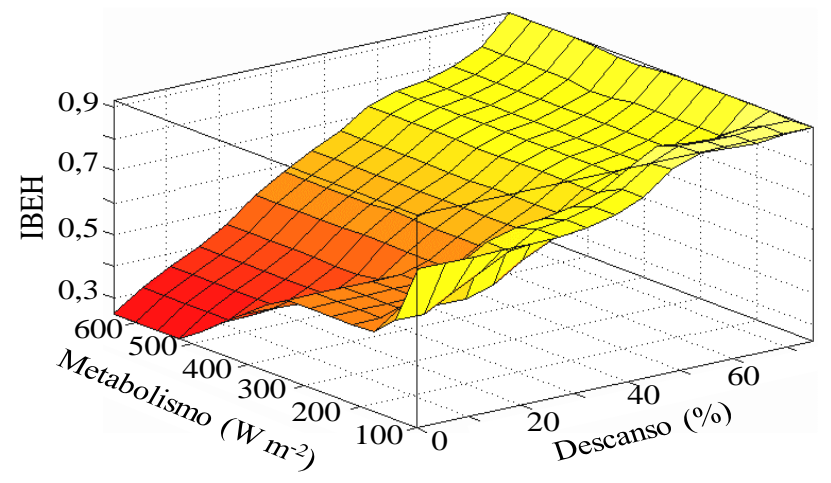

B

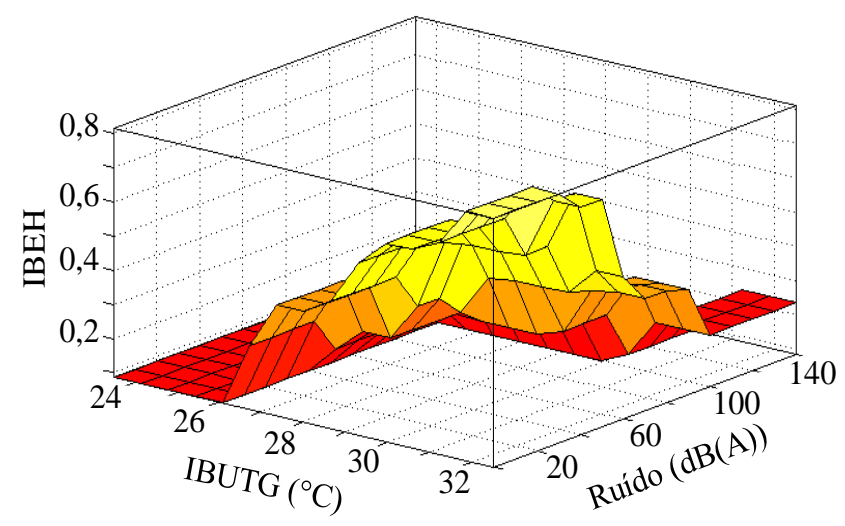

$\mathrm{D}$

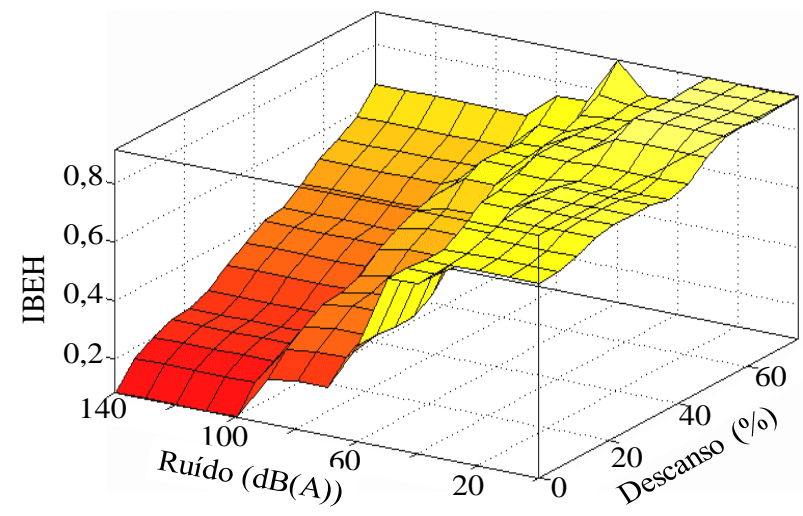

$\mathrm{F}$

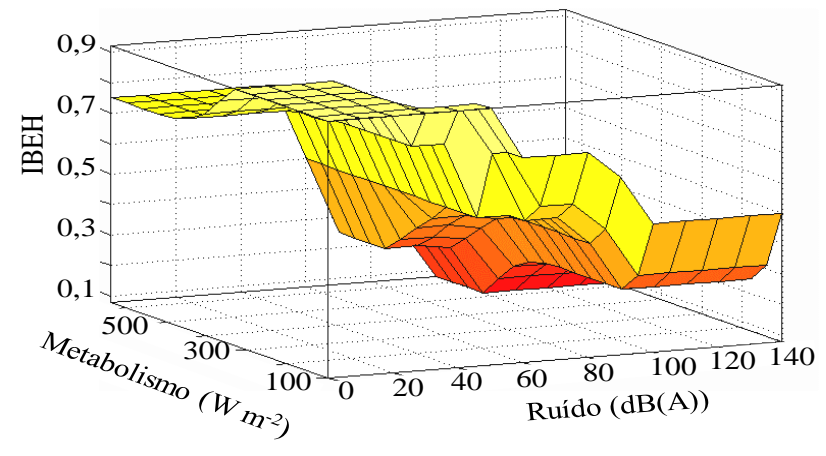

FIGURA 4. IBEH simulado em função das variáveis de entrada: A - IBUTG $\left({ }^{\circ} \mathrm{C}\right)$ e descanso $(\%)$; B - IBUTG $\left({ }^{\circ} \mathrm{C}\right)$ e nível de ruído (dBA); $\mathrm{C}$ - metabolismo $\left(\mathrm{W} \mathrm{m}{ }^{-2}\right)$ e IBUTG $\left({ }^{\circ} \mathrm{C}\right)$; D nível de ruído (dBA) e descanso (\%); E - metabolismo $\left(\mathrm{W} \mathrm{m}^{-2}\right)$ e descanso $(\%)$, e $\mathrm{F}$ metabolismo $\left(\mathrm{W} \mathrm{m}^{-2}\right)$ e nível de ruído (dBA). HWBI simulated as a function of combinations of the input variables: A - WBGTI $\left({ }^{\circ} \mathrm{C}\right)$ and rest time $(\%), \mathrm{B}$ WBGTI $\left({ }^{\circ} \mathrm{C}\right)$ and noise level (dBA), C - metabolic rate $\left(\mathrm{W} \mathrm{m}^{-2}\right)$ and WBGTI $\left({ }^{\circ} \mathrm{C}\right)$, $\mathrm{D}$ - noise level (dBA) and rest time $(\%), \mathrm{E}$ - metabolic rate $\left(\mathrm{W} \mathbf{m}^{-2}\right)$ and rest time (\%) and $\mathrm{F}$ - metabolic rate $\left(\mathrm{W} \mathrm{m}^{-2}\right)$ and noise level (dBA).

Podem-se observar pelas simulações os pontos em que o IBEH é alto, evidenciando ambientes onde a situação laboral do trabalhador se encontra em conforto, fazendo com que toda sua atenção esteja voltada para o exercício da atividade.

Os pontos onde o IBEH é baixo estão diretamente relacionados a ambientes insalubres, fazendo com que ocorra maior desgaste físico e psicológico do trabalhador e aumentando assim as chances de erros e riscos de acidentes de trabalho. De posse dessas simulações, pode-se inferir 
sobre a salubridade do ambiente de trabalho e propor medidas de controle, visando ao conforto do trabalhador. Tais medidas podem ser: aumento do tempo de descanso; manejo do horário de trabalho, dando preferência para as horas do dia em que a temperatura está mais baixa; uso de roupas apropriadas para diminuir a sensação térmica e aumentar a reflexão da radiação solar, além do uso de protetores auriculares. O aumento do tempo de descanso como forma de reduzir o efeito do ruído e de temperatura sobre humanos foi reportado por GOSLING \& ARAÚJO (2008).

\section{Teste do modelo fuzzy}

Para testar o modelo fuzzy, foram utilizados dados experimentais de IBUTG médio durante o dia de coleta, em cada equipamento (trator $-21,8{ }^{\circ} \mathrm{C}$, motosserra $-20,9{ }^{\circ} \mathrm{C}$, roçadora $-22,6{ }^{\circ} \mathrm{C}$ e derriçadora $-22,1^{\circ} \mathrm{C}$ ), e nível de ruído médio para cada distância de afastamento, sendo simulado o tempo de descanso ideal para cada máquina (Tabela 4), tendo como variáveis predefinidas do sistema o índice de bem-estar humano médio para atividade pesada com consumo metabólico de $400 \mathrm{~W} \mathrm{~m}^{-2}$ (GOSLING \& ARAÚJO, 2008).

TABELA 4. Predição fuzzy para o tempo de descanso nos equipamentos avaliados com diferentes distâncias de coletas. Fuzzy prediction for the rest time on equipment evaluated with distinct distance of data recording.

\begin{tabular}{|c|c|c|c|c|c|c|c|c|c|c|c|c|}
\hline \multirow{2}{*}{ Equipamento } & & \multicolumn{11}{|c|}{ Distância da Fonte Ruidosa (m) } \\
\hline & & 0 & 2 & 4 & 6 & 8 & 10 & 12 & 14 & 16 & 18 & 20 \\
\hline \multirow[b]{2}{*}{ Trator } & $\mathrm{JR}^{1}$ & 94,3 & 8,7 & 35,0 & 82,9 & 79,9 & 78,5 & 77,5 & 75,9 & 75,0 & 74,2 & 73,6 \\
\hline & $\mathrm{TD}^{2}$ & 64,0 & 40,6 & 39,0 & 39,0 & 39,0 & 39,0 & 39,0 & 39,0 & 39,0 & 39,0 & 39,0 \\
\hline \multirow[b]{2}{*}{ otc } & $\mathrm{NR}^{1}$ & 106,3 & 98,0 & 93,9 & 92,2 & 89,3 & 88,2 & 86,8 & 85,0 & 83,7 & 82,8 & 81,4 \\
\hline & $\mathrm{TD}^{2}$ &, 2 & 54,1 & 64,0 & 62,0 & 40,8 & 40,3 & 39,5 & 39,0 & 39,0 & 39,0 & 39,0 \\
\hline \multirow[b]{2}{*}{ Roça } & $\mathrm{NR}^{1}$ & 103,2 & 96,7 & 92,1 & 88,7 & 85,5 & 84,0 & 82,6 & 81,5 & 81,2 & 80,0 & 79,4 \\
\hline & $\mathrm{TD}^{2}$ & 64,1 & 64,1 & 61,8 & 40,6 & 39,2 & 39,0 & 39,0 & 39,0 & 39,0 & 39,0 & 39,0 \\
\hline \multirow{2}{*}{ Derriçadora } & $\mathrm{NR}^{1}$ & 84,3 & 78,8 & 75,4 & 71,3 & 68,4 & 65,7 & 63,4 & 62,2 & 61,2 & 60,1 & 59,3 \\
\hline & $\mathrm{TD}^{2}$ & 39,0 & 39,0 & 39,0 & 39,0 & 39,0 & 39,0 & 33,5 & 30,5 & 28,0 & 25,3 & 25,0 \\
\hline
\end{tabular}

$\mathrm{NR}^{1}$ - Nível de ruído (dB(A)); $\mathrm{TD}^{2}$ - Tempo de descanso (\%)

De acordo com os resultados obtidos na Tabela 6, pode-se observar que, para os sistemas avaliados, a modelagem fuzzy apresentou tempo de descanso ideal para cada equipamento e distância de trabalho, variando de $64,2 \%$ (motosserra, próximo ao ouvido do operador) até $25 \%$ (derriçadora, $20 \mathrm{~m}$ de distância do operador).

Em pesquisa avaliando a eficiência de redução do ruído com protetores auriculares do tipo concha, NIELSEN (2001) obteve valores de atenuação de 16,2 dB(A). Utilizando essa informação para simular o modelo fuzzy nos equipamentos avaliados com o uso de protetores auriculares pelos trabalhadores, os resultados obtidos para o tempo de descanso foram 35,8\% para o maior nível de ruído encontrado, $90,1 \mathrm{~dB}(\mathrm{~A})$, com a motosserra próximo ao ouvido do operador, e $0 \%$ para o menor nível de ruído observado nas coletas, $43,1 \mathrm{~dB}(\mathrm{~A})$, com a derriçadora a $20 \mathrm{~m}$ de distância do operador, ou seja, essa atividade pode ser executada de forma salubre sem a necessidade de descanso para o trabalhador.

Embora em alguns casos o nível de ruído obtido nas coletas apresente valores abaixo do limite máximo permitido, o tempo de descanso simulado foi alto devido à alta taxa de metabolismo das atividades desenvolvidas. Estes resultados mostram a capacidade do modelo fuzzy em quantificar a interação entre as variáveis estudadas, ponderando seus valores de acordo com as regras preestabelecidas, comprovando assim sua eficácia em classificar os diferentes ambientes de trabalho.

Visando a ilustrar a diferença de classificação dos resultados da modelagem fuzzy, foram desenvolvidos gráficos (Figura 5) utilizando os dados de campo de nível de ruído e IBUTG, e simulado o tempo de descanso ideal em cada equipamento, para todas as distâncias observadas. 
A

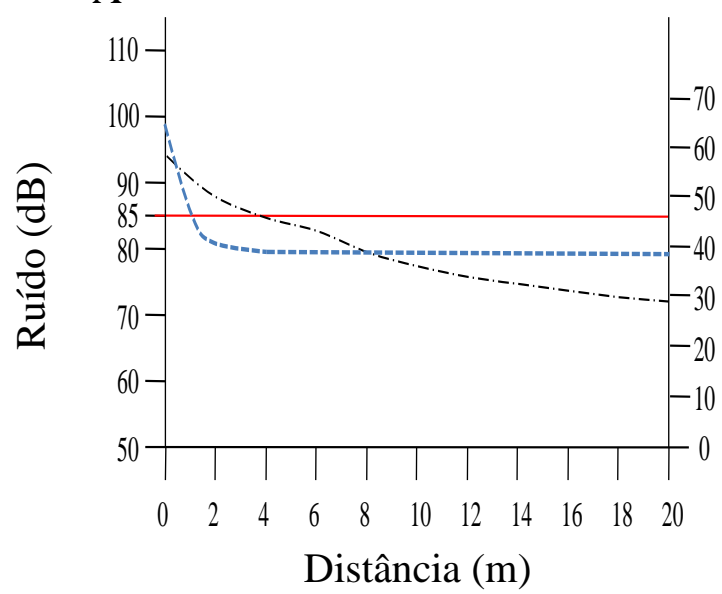

$\mathrm{C}$

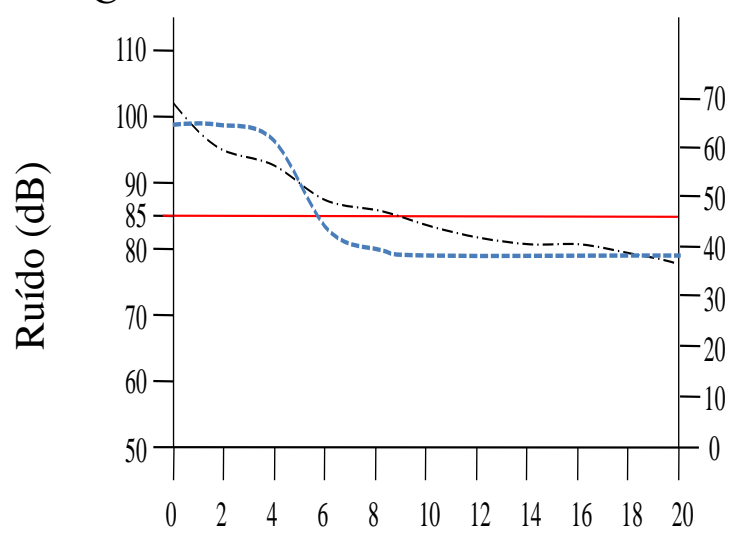

Distância (m)

-.-.- Ruído -------Descanso

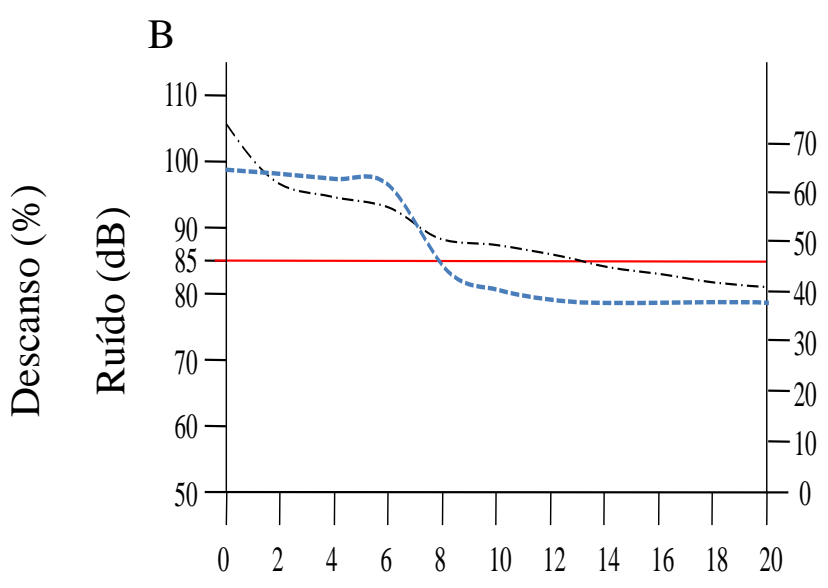

Distância (m)

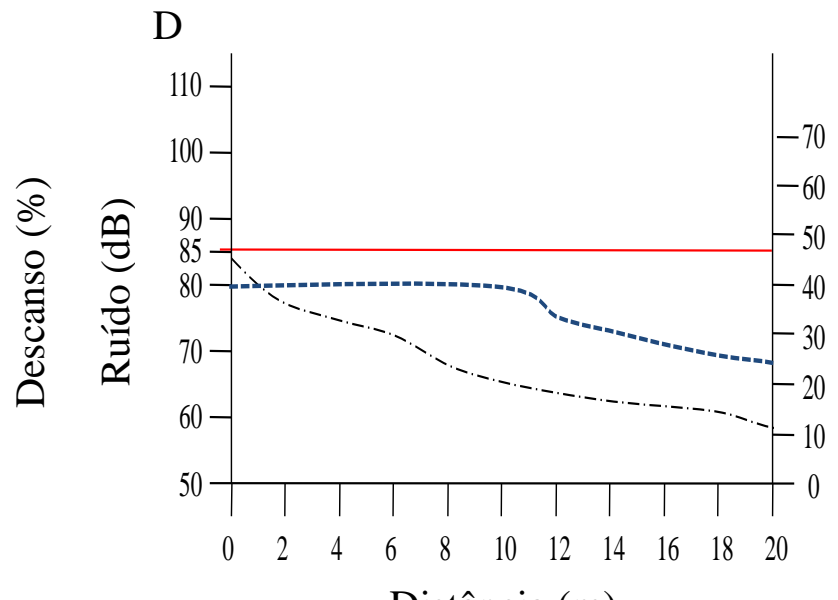

Distância (m)

Limite de conforto para ruído (NR-15)

FIGURA 5. Comparação dos dados de ruído com os valores de tempo de descanso simulado e limite máximo de ruído permitido pela norma para A - trator; B - motosserra; C roçadora, e D - derriçadora. Comparison of noise data with values of the simulated rest time and maximum noise level allowed by the standard for $\mathrm{A}$ - tractor, $\mathrm{B}$ chainsaw, $\mathrm{C}$ - brushcutter and $\mathrm{D}$ - harvester.

Dos equipamentos avaliados somente a derriçadora apresentou níveis de ruído abaixo do limite estabelecido em norma para todas as distâncias de coleta, sendo que o tempo de descanso simulado foi o menor entre todos os equipamentos avaliados. Embora o nível de ruído nesse equipamento apresente valores dentro dos limites estabelecidos em norma, valores de ruído acima de $55 \mathrm{~dB}(\mathrm{~A})$ causam comprometimentos orgânicos, como estresse e desconforto (OMS, 2001).

Diante desses resultados, pode-se inferir sobre a distância segura e o tempo de descanso ideal para que o trabalhador se encontre em melhores condições de bem-estar em determinado equipamento. Nos casos em que não é possível realizar o trabalho a uma distância segura ou realizar intervalos regulares de descanso, faz-se então necessário o uso de equipamentos de segurança e proteção individual, tais como roupas adequadas para reduzir os efeitos da radiação solar e o uso de protetores auriculares.

Ressalta-se que o modelo fuzzy proposto pode ser usado para quantificar o bem estar humano em quaisquer ambientes de trabalho nos quais o trabalhador esteja sujeito às variáveis de entrada considerada (IBUTG, nível de ruído, metabolismo e tempo de descanso). No caso específico dos dados usados para o teste do modelo, pode-se inferir tanto sobre o nível de salubridade a que o operador está sujeito, quanto no seu entorno. 


\section{CONCLUSÕES}

O modelo fuzzy proposto permite estimar o índice de salubridade para trabalhadores, com base nas normativas de bem-estar vigente, considerando a influência de cada variável de entrada ao homem, podendo ainda auxiliar na tomada de decisões no controle do ambiente de trabalho, evitando assim perdas de produtividade e danos à saúde do trabalhador.

\section{AGRADECIMENTOS}

Os autores expressam seus agradecimentos à CAPES, CNPq e FAPEMIG, pelo suporte financeiro a esta pesquisa.

\section{REFERÊNCIAS}

ABNT. ASSOCIAÇÃO BRASILEIRA DE NORMAS TÉCNICAS. Norma NBR 9999: Medição do nível de ruído no posto de operação de tratores e máquinas agrícolas. Rio de Janeiro, 1987. 21 p.

ALMEIDA, C. F. M.; KAGAN, N. Aplicação de algoritmos genéticos e teoria dos conjuntos fuzzy no dimensionamento de sistemas de monitoração para redes de transmissão de energia elétrica. Revista Controle \& Automação, Campinas, v.21, n.4, p.363-378, 2010.

AMENDOLA, M.; SOUZA, A.L.; BARROS, L.C. Manual do uso da teoria dos conjuntos fuzzy no MATLAB 6.5. Campinas: FEAGRI \& IMECC/ UNICAMP, 2005. v.2, 44 p.

AYYUB, B.M.; KLIR, G.J. Uncertainty modeling and analysis in engineering and the sciences. Boca Raton: Chapman \& Hall/CRC, 2006. 378 p.

BAESSO, M.M.; TEIXEIRA, M.M.; JUNIOR, F.A.R.; JUNIOR, R.G.M.; FERNANDES, H.C. Avaliação do nível de ruído emitido por um conjunto trator-pulverizador com e sem assistência de ar. Revista Engenharia na Agricultura, Viçosa-MG, v.16, n.4, p.400-407, 2008.

BARIN, A.; CANHA, L.N.; MAGNANO, K.F.; ABAIDE, A.R. Seleção de fontes alternativas de geração distribuída utilizando uma análise multicriterial baseada no método AHP e na lógica fuzzy. Revista Controle \& Automação, Campinas, v.21, n.5, p.477-486, 2010.

BRUNASSI, L.A.; MOURA, D.J.; NÄÄS, I.A.; VALE, M.M.; SOUZA, S.R.L. Improving detection of dairy cow estrus using fuzzy logic. Scientia Agricola, Piracicaba, v.67, n.5, p.503-509, 2010 .

CARVALHO, C.C.S. Avaliação ergonômica em operações do sistema produtivo de carne de frango. 2009. 163 f. Tese (Doutorado em Engenharia Agrícola) - Universidade Federal de Viçosa, Viçosa-MG, 2009.

CORNELISSEN, A.M.G.; VAN DEN BERG, J.; KOOPS, W.J.; KAYMAK, U. Eliciting expert knowledge for fuzzy evaluation of agricultural production systems. Rotterdam: Erasmus Research Institute of Management, 2002. (Report, ERS-2002-108-LIS). Disponivel em: <http://papers.ssrn.com/sol3/papers.cfm?abstract_id=371055>. Acesso em: 20 dez. 2011.

COUTO, H.A. Temas de saúde ocupacional - coletânea dos cadernos da Ergo. Belo Horizonte: Ergo, 1987. $250 \mathrm{p}$.

CREMASCO, C.P.; GABRIEL FILHO, L.R.A.; CATANEO, A. Metodologia de determinação de funções de pertinência de controladores fuzzy para a avaliação energética de empresas de avicultura de postura. Revista Energia na Agricultura, Botucatu, v.25, n.1, p.21-39, 2010.

CUNHA, J.P. A.R.; TEODORO, R.E.F. Avaliação do nível de ruído em derriçadores e pulverizadores motorizados portáteis utilizados em lavouras de café. Bioscience Journal, Uberlândia, v.22, n.3, p.71-77, 2006.

ESCOBAR, C.; GALINDO, J. Fuzzy control in agriculture: simulation software. In: INDUSTRIAL SIMULATION CONFERENCE, 2004, Malaga. p.45-49. 
FERREIRA, L.; YANAGI JUNIOR, T.; NÄÄS, I.A.; LOPES, M.A. Development of algorithm using fuzzy logic to predict estrus in dairy cows: Part I. Agricultural Engineering International: The CIGR Ejournal, Beijing, v.9, p.1-16, 2007.

GOSLING, M.; ARAÚJO, G.C.D. Saúde física do trabalhador rural submetido a ruídos e à carga térmica: um estudo em operadores de tratores. Revista O Mundo da Saúde, São Paulo, v.32, n.3, p.275-286, 2008.

LEITE, M. S.; FILETI, A. M. F.; SILVA, F. V. Desenvolvimento e aplicação experimental de controladores fuzzy e convencional em um bioprocesso. Revista Controle \& Automação, Campinas, v.21, n.2, p.147-158, 2010.

MARTINO, J.P. Technological forecasting for decision making. New York: Elsewier, 1983. 386 p. MATLAB 6.5. Disponível em: www.ime.unicamp.br/ laeciocb/MANUAL_2005.pdf/. 2005. 46 p.

MION, R.L.; VILIOTTI, C. A.; DANTAS, M.J.F.; NASCIMENTO, E.M.S. Avaliação dos níveis de ruído de um conjunto mecanizado trator e semeadora adubadora pneumática. Revista Engenharia na Agricultura, Viçosa-MG, v.17, n.2, p.87-92, 2009.

MTE. MINISTÉRIO DO TRABALHO E EMPREGO. Portaria 3.214 de jul. 1990. Normas regulamentadoras de segurança e saúde no trabalho (NR-15): atividades e operações insalubres. Brasília, 1990. Disponível em:

<http://www.mte.gov.br/temas/segsau/legislacao/normas/conteudo/nr15>. Acesso em: set. 2007.

NÄÄS, I.A.; ROMANINI, C.E.B.; SALGADO, D.D.; LIMA, K.A.O.; VALE, M.M. Impact of global warming on beef cattle production cost in Brazil. Scientia Agricola, Piracicaba, v.67, n.1, p.1-8, 2010.

NIELSEN, R.M. Comportamento de três protetores auriculares tipo concha, em ambientes com ruídos em baixa frequência. 2001. 58 f. Dissertação (Mestrado em Engenharia de Produção) Universidade Federal do Rio Grande do Sul, Porto Alegre. 2001.

OMS. ORGANIZAÇÃO MUNDIAL DA SAÚDE. Occupational and community noise. Geneva: World Health Organization. 2001. Disponível em:

<http://www.who.int/mediacentre/factsheets/fs258/en/>. Acesso em: 20 fev. 2002.

OWADA, A. N.; NÄ̈̈S, I. A.; MOURA, D. J.; BARACHO, M. S. Estimativa do bem-estar de frangos de corte em função da concentração de amônia e grau de luminosidade no galpão de produção. Engenharia Agrícola, Jaboticabal, v.27, n.3, p.611-618, set./dez.2007.

PANDORFI, H.; SILVA, I.J.O.; GUISELINI, C.; PIEDADE, S.M.S. Uso da lógica fuzzy na caracterização do ambiente produtivo para matrizes gestantes. Engenharia Agrícola, Jaboticabal, v.27, n.1, p.83-92, 2007.

PEREIRA, D.F.; BIGHI, C.A.; FILHO, L.R.G.; GABRIEL, C.P.C. Sistema fuzzy para estimativa do bem-estar de matrizes pesadas. Engenharia Agricola, Jaboticabal, v.28, n.4, out./dez. 2008.

Disponível em: http://www.scielo.br/scielo.php?script=sci_arttext\&pid=S010069162008000400002\&lng=pt\&nrm=iso. Acesso em: 20 jan. 2011.

PREBLE, J. F. The selection of Delphi panels for strategic planning purposes. Strategic Management Journal, Sussex, v.5, n.2, p.157-170,1984.

ROBIN, P. Segurança e ergonomia em maquinaria agrícola. São Paulo: IPT, 1987. 24 p.

SCHIASSI, L.; YANAGI JÚNIOR, T.; FERREIRA, L.; DAMASCENO, F. A.; YANAGI, S.N.M. Metodologia fuzzy aplicada à avaliação do aumento da temperatura corporal em frangos de corte. Engenharia na agricultura, Viçosa-MG, v.16, n.2, p.181-191, 2008. 
SILVEIRA, G.P.; VENDITE, L.L.; BARROS, L.C. Software desenvolvido a partir de um Modelo Matemático Fuzzy para predizer o estágio patológico do Câncer de Próstata. Biomatemática, Campinas, v. 18, p.27-36, 2008.

SIVANANDAM, S. N.; SUMATHI, S.; DEEPA, S. N. Introduction to fuzzy logic using MATLAB. Berlin: Springer, 2007. 430 p.

TAYLOR, J. Delphi method applied to tourism. In: Wittis, M. L. Tourism marketing and management handbook. New York: Prentice Hall, 1988, p.95-99. 